\title{
NEUROPSYCHOPHARMACOLOGY REVIEWS Sleep therapeutics and neuropsychiatric illness
}

\author{
Andrew D. Krystal ${ }^{1}$
}

\begin{abstract}
Alterations in sleep are extremely common in patients with neuropsychiatric illness. In addition, sleep disorders such as insomnia, obstructive sleep apnea, rapid eye movement sleep behavior disorder, and circadian rhythm disorders commonly occur at a rate greater than the general population in neuropsychiatric conditions. Historically, sleep problems have been viewed as symptoms of associated neuropsychiatric disorders. However, there is increasing evidence suggesting a complex inter-relationship with possible bidirectional causality. The inter-relatedness of these conditions represents an opportunity for understanding mechanisms and improving clinical treatment. To the extent that sleep problems affect neuropsychiatric conditions, it may be possible to address sleep problems and have a positive impact on the course of neuropsychiatric illnesses. Further, some treatments for sleep disorders have direct effects on neuropsychiatric illnesses that may be unrelated to their effects on sleep disorders. Similarly, neuropsychiatric conditions and their treatments can affect sleep and sleep disorders. This article reviews available evidence on the effects of therapies for sleep disorders on neuropsychiatric conditions and also secondarily considers the impacts of therapies for neuropsychiatric conditions on sleep. Primary goals of this review are to identify gaps in current research, to determine the extent to which the cross-therapeutic effects of these treatments help to elucidate therapeutic or pathological mechanisms, and to assist clinicians in optimizing therapeutic choice in patients with sleep disorders and neuropsychiatric conditions.
\end{abstract}

Neuropsychopharmacology (2020) 45:166-175; https://doi.org/10.1038/s41386-019-0474-9

\section{INTRODUCTION}

Alterations in sleep are extremely common in patients with neuropsychiatric illness. These alterations include disturbances of sleep, a change in the distribution of sleep stages across the night, parasomnias (e.g., nightmares), and excessive daytime sleepiness $[1,2]$. In addition, sleep disorders commonly occur at a rate greater than the general population in neuropsychiatric conditions [3-5]. Such sleep disorders include insomnia, obstructive sleep apnea (OSA), rapid eye movement (REM) sleep behavior disorder, and circadian rhythm disorders.

Historically, sleep problems have been viewed as symptoms of associated neuropsychiatric disorders. However, there is increasing evidence suggesting a complex inter-relationship with possible bidirectional causality $[2,6]$. The inter-relatedness of these conditions represents an opportunity to understand mechanisms and for improving clinical treatment.

To the extent that sleep problems affect neuropsychiatric conditions, it may be possible to address sleep problems and have a positive impact on the course of neuropsychiatric illnesses. Further, some treatments for sleep disorders have direct effects on neuropsychiatric illnesses that may be unrelated to their effects on sleep disorders. An example would be the use of clonazepam to treat REM sleep behavior disorder [7]. Clonazepam is a medication with direct anxiety-reducing effects that are independent of its effects on REM sleep behavior disorder [8].

Similarly, neuropsychiatric conditions and their treatments can affect sleep and sleep disorders. This also provides opportunities for optimizing clinical therapy. Improving neuropsychiatric conditions can have an ameliorative effect on the associated sleep alterations $[9,10]$. At the same time, treatments for neuropsychiatric conditions can have effects on sleep that are independent of the effects on those neuropsychiatric conditions. Such effects may be therapeutic as in the sleepenhancing effects of some antipsychotic medication when these agents are used to treat individuals with disturbed sleep [11]. These effects may also have a negative impact on sleep, such as the sleep disturbance that occurs in $\sim 4-23 \%$ of individuals treated with selective serotonin reuptake inhibitors (SSRIs), serotonin norepinephrine reuptake inhibitors (SNRIs), and bupropion for major depressive disorder (MDD) $[12,13]$. Optimizing choice of therapy for neuropsychiatric conditions includes taking into account such effects on sleep.

This article reviews available evidence on the effects of therapies for sleep disorders on neuropsychiatric conditions and also secondarily considers the impacts of therapies for neuropsychiatric conditions on sleep. Primary goals of this review are to identify gaps in current research, to determine the extent to which the cross-therapeutic effects of these treatments help to elucidate therapeutic or pathological mechanisms, and to assist clinicians in optimizing therapeutic choice in patients with sleep disorders and neuropsychiatric conditions. It is not possible to cover all sleep disorders and neuropsychiatric conditions in this article. As a result, we focus on the subset where the links with sleep and neuropsychiatric therapies are best characterized (MDD, bipolar disorder, anxiety disorders, post-traumatic stress disorder (PTSD), substance use disorders, and schizophrenia) and the sleep disorders with which these links most commonly occur (insomnia, OSA, restless legs syndrome (RLS), parasomnias, and circadian rhythm disorders).

${ }^{1}$ University of California San Francisco, San Francisco, CA, USA
Correspondence: Andrew D. Krystal (andrew.krystal@ucsf.edu)

Received: 2 May 2019 Revised: 23 July 2019 Accepted: 30 July 2019

Published online: 3 August 2019 


\section{NEUROPSYCHIATRIC CONDITIONS AND IMPACTS OF TREATMENTS ON SLEEP}

This section consists of a brief overview of a number of neuropsychiatric conditions where there are significant relationships to sleep and sleep therapeutics and there is evidence that therapies for these conditions impact sleep/wake function. These include MDD, bipolar disorder, anxiety disorders, PTSD, substance use disorders, schizophrenia. We also note where there are effects of treatments for these neuropsychiatric conditions, which impact sleep and sleep disorders.

\section{Major depressive disorder}

MDD has a population lifetime prevalence of $\sim 10 \%$ and is defined by the presence of at least 5 of a constellation of symptoms for at least 2 weeks [14-17]. These symptoms include depressed mood, anhedonia, appetite or weight changes sleep difficulties, psychomotor agitation or retardation, fatigue or loss of energy, diminished ability to think or concentrate, feelings of worthlessness or excessive guilt, and suicidality. However, one of the symptoms must be either depressed mood or anhedonia. Either insomnia or hypersomnia are reported by $\sim 75 \%$ of those with this condition $[18,19]$ and polysomnographic findings in MDD include evidence of sleep disturbance, decreased amount and percentage of N3 sleep, diminished electroencephalogram (EEG) slow-wave activity, decreased REM latency (time from sleep onset to onset of REM), increased percentage of REM sleep, and increased REM density (number of eye movements per minute of REM sleep) [20-23].

There is evidence of complex causality between MDD and the co-occurring sleep problems. Insomnia often precedes the onset of MDD [24] and many studies indicate that having insomnia significantly increases the risk of developing MDD in the future [25].

In terms of other associations between sleep and MDD, a significantly elevated rate of OSA is seen in those with MDD. It is estimated that $20 \%$ of those with MDD have OSA [26]. The cause for this elevated frequency of OSA in MDD is unknown. There is no clear indication of an association of MDD with sleep disorders other than insomnia and OSA.

Lastly, treatments for MDD can have significant impacts on sleep/wake function. As mentioned in the introduction, sleep disturbance occurs in 4-23\% treated with the standard, first-line therapies for this condition, including SSRIs, SNRIs, and bupropion $[12,13]$. At the same time, SSRIs and SNRIs are associated with an increased rate of somnolence (5-25\%) [12]. Antidepressants have also been associated with causing or exacerbating RLS and periodic limb movements of sleep (PLMS) [27-29]. It has been reported that a subset of antidepressants are more likely to be associated with RLS (mirtazapine) and PLMS (sertraline, fluoxetine, and amitriptyline) $[27,29]$. There is also evidence that antidepressants can be associated with REM sleep without atonia in individuals both with and without REM sleep behavior disorder $[30,31]$.

\section{Bipolar disorders}

The bipolar disorders consist of: bipolar I disorder, which includes a history of at least one manic episode (a period of at least 1 week of elevated, expansive or irritable mood, and at least three of the following: grandiosity, decreased sleep need, rapid/pressured speech, increase in goal-directed activities, increased distractibility, increased risky behavior, or racing thoughts), bipolar II disorder marked by at least one major depressive episode and at least one episode of hypomania (a minimum of 4 days of the symptoms of mania, but without psychosis and without the degree to severity which causes significant impairment in functioning or requires hospitalization), and cyclothymic disorder where affected individuals experience sub-syndromal symptoms of hypomanic and depressive episodes for at least half of a 2-year period with no symptom-free periods of more than 2 months [14].
The lifetime prevalence of the bipolar disorders in the general population is $2.6-6.5 \%$ [32].

Decreased need for sleep is one of the cardinal symptoms of mania and, while depressed, nearly all patients with bipolar disorders report insomnia or hypersomnia [33]. Further, sleep problems are often present in the period between mood episodes, with the majority experiencing insomnia [34]. Polysomnographic findings in bipolar disorders are similar to what is seen with unipolar MDD, including evidence of sleep disturbance, shortened REM latency, and increased REM density $[35,36]$.

As with MDD there is evidence of bi-directionality among sleep problems and bipolar disorders. Sleep disturbance can precede the onset of episodes of mood symptoms of bipolar disorder by a significant period of time [37]. Further, experimental sleep deprivation of patients with bipolar disorders often precipitates mania [38].

Treatments for bipolar disorders can also have effects on sleep. The same medications used to treat unipolar MDD are often used to treat bipolar depression with the recommendation that they be given along with mood-stabilizing medication. The sleep impacts of these medications were described in the prior section. Other agents used to treat bipolar depression include lamotrigine, which can disturb sleep in some patients and medications used to treat mania or as general mood stabilizers, which includes atypical antipsychotic medication, lithium, divalproex sodium, and carbamazepine. All of the anti-manic agents and mood stabilizers tend to promote sleep and can cause daytime sedation [39]. These medications can also cause weight gain, which could precipitate or exacerbate OSA [40]. The antipsychotic medications can also cause or exacerbate RLS [41].

\section{Anxiety disorders}

Here, we focus on one of the anxiety disorders, generalized anxiety disorder (GAD) because this is the anxiety disorder where the links with sleep have been best established. GAD is defined as excessive anxiety/worry about number of things for the majority of a period of at least 6 months accompanied by at least 3 of the following: feeling restless, easy fatigability, difficult concentrating, irritability, muscle tension, or sleep disturbance [14]. The lifetime general population prevalence of GAD is $9 \%$ and it is twice as common in women than in men $[42,43]$. Problems both falling and staying asleep occur in over $50 \%$ of individuals with this disorder [44].

Medications used to treat GAD can have important effects on sleep. First-line pharmacologic treatments for GAD are SSRIs and SNRIs, which can be associated with both insomnia and reported daytime sleepiness as described above [45]. Second-line treatments consist of pregabalin, buspirone, and benzodiazepines, all of which are sleep enhancing and can be beneficial for sleep disturbance when taken at bedtime, but associated with daytime sleepiness when taken during the day [45].

Post-traumatic stress disorder

PTSD is defined by an exposure to actual or threatened death, serious injury, or sexual violence followed by: at least one symptom of intrusion of the trauma (experiencing recurrent intrusive memories of the trauma, recurring related nightmares, flashbacks, or marked physiologic reactions to reminders of the trauma); avoidance of memories, thoughts, or feelings related to the event, or anything that triggers such experiences; at least two negative alterations in cognition or mood (amnesia for an aspect of the event, negative beliefs or expectations about oneself, others, or the world, blaming self or others for the cause or consequences of the event, persistent negative emotions, diminished interest in activities, feelings of detachment from others, or inability to experience positive emotions); and at least 2 alterations in arousal and reactivity (irritability, reckless/selfdestructive behavior, hypervigilance, exaggerated startle 
168

response, concentrations problems, or sleep disturbance) [14]. The general population lifetime prevalence of PTSD is estimated to be $8.7 \%$ [46].

Two types of sleep problems are strongly associated with PTSD: nightmares and sleep disturbance. Sixty to $90 \%$ of PTSD patients experience problems with sleep onset or maintenance and this is the most common symptom of combat-related PTSD [47, 48]. A contributing factor to the sleep disturbance can be fear of going to sleep, which can emerge due to experiencing memories of the traumatic event when trying to sleep and due to nightmares [49]. Polysomnographic findings in those with PTSD include fragmentation of REM into a larger number of shorter periods, which has been interpreted as evidence of the importance of REM-related pathology $[50,51]$.

There is some data which suggests that the relationship between sleep problems and PTSD may be complex. In a study of military personnel, those who had insomnia prior to being deployed had a greater rate of developing PTSD when evaluated post-deployment [48, 52]. There is also a report that REM fragmentation post trauma might mediate the development of PTSD symptoms [53]. Some data also suggest that sleep problems mediate the relationship between traumatic brain injury (TBI) and the development of symptoms including PTSD symptoms [54].

PTSD is also linked with other sleep pathology. Individuals with this condition are at increased risk of having OSA [55]. Individuals with PTSD have also been reported to have REM sleep without atonia, which has features that overlap with REM behavior disorder, but likely is a distinct entity [56].

There are no pharmacologic therapies with an evidence base indicating compelling therapeutic effects on PTSD. First-line therapy consists of SSRIs and SNRIs, although the only agents Food Drug Administration (FDA) approved for the treatment of this condition are sertraline and paroxetine [57]. As such, like MDD and GAD, treatment brings with it a risk of insomnia, daytime sedation, PLMs, and REM sleep without atonia. Prazosin is the only agent with evidence of efficacy for the treatment of nightmares, although a recent large multi-site study calls that into question [58]. This medication has been reported to increase sleep time, increase REM time, and increase REM period duration, and the REM-related changes have been speculated to be linked to its therapeutic effects on nightmares [59].

Substance use disorders

Substance use disorders encompass problematic use of 10 types of substances [14]. We address only alcohol use disorder as this is where the links with sleep have been best studied. Alcohol use disorder is defined as a pattern of alcohol use for a 12-month period associated with significant impairment or distress as reflected in two or more of the following: intake in greater quantities or duration than intended; desire to limit use; devoting significant time to obtaining, using, or recovering from use of alcohol; experiencing craving to drink; drinking causing an inability to fulfill major role obligations; continued use despite use causing problems; giving up or limiting activities because of drinking; drinking in situations where it is dangerous to do so; continued use in the face of a persistent alcohol-related problem; the development of tolerance (either a need to increase amount used to achieve a given effect or diminished effect with continued use of the same amount of alcohol); or the development of withdrawal symptoms on discontinuation [14,60].

Those with alcohol use disorder experience difficulty falling asleep, staying asleep, daytime sleepiness, and parasomnias [61-65]. Further, during periods of heavy drinking individuals can lose circadian rhythmicity of sleep/wake function and develop brief periods of sleep throughout the $24 \mathrm{~h}$ period [64]. The potential for alcohol to induce brief periods of sleep whenever drinking occurs is thought to contribute to the development of this pattern. The initial phase after discontinuation of regular alcohol use is associated with disturbed sleep and N3 reduction and disruption of REM and increased amount of REM is evident on polysomnography [64]. Complaints of sleep difficulties and polysomnographic evidence for disturbance of sleep and increased REM are reported to continue for up to 3 years $[64,66]$.

Evidence of complex causality of sleep problems and alcohol use is that the post-discontinuation sleep of those with alcohol use disorder predicts the likelihood of relapse to drinking $[67,68]$. In this regard, individuals with alcohol use disorder in recovery given the opportunity to drink alcohol, who report sleep difficulties, are more likely to choose to consume alcohol [69].

Alcohol use can also impact sleep disorders. Because alcohol has respiratory depressant and myorelaxant effects it can exacerbate breathing-related sleep disorders [70, 71]. Alcohol use disorder has been hypothesized to be indirectly associated with RLS and PLMS because it can lead to low ferritin, low magnesium. and vitamin B12 deficiency [64].

\section{Schizophrenia}

Schizophrenia is defined by at least 6 months of difficulties within which there is at least a month of persistent delusions, hallucinations, or disorganized speech, at least two of a set of five features (delusions, hallucinations, disorganized speech, disorganized or catatonic behavior, or negative symptoms like blunted emotional expression), and impairment in at least one major area of function (work, interpersonal relations, self-care, etc.). [14] The lifetime prevalence of schizophrenia is $0.5-1 \%$ [72].

Sleep problems are not a core symptom of schizophrenia; however, up to $80 \%$ of those with with schizophrenia suffer from disturbance of sleep [73]. Individuals with schizophrenia have been found to have increased sleep latency, awakenings, and naps, and decreased sleep efficiency [74, 75]. Alterations in circadian rhythm marked by the tendency to be awake at night and asleep during the day are also common [76]. Polysomnographic studies consistently demonstrate disturbed sleep in acutely psychotic patients with schizophrenia [77]. The most consistent sleep stage-related finding appears to be diminished N3 sleep, which has been observed in antipsychotic naive patients and in first-degree relatives of those with schizophrenia [78, 79]. Further, lower non-REM sleep EEG slow-wave amplitude has been reported to be correlated with greater deficit symptom severity $[80,81]$. There are also reports that greater severity of delusions, hallucinations, and disorganized thought is correlated with decreased sleep efficiency, longer sleep onset latency, and shorter REM latency [82, 83].

Those with schizophrenia have also been found to have a decrease in sleep spindles $(14-16 \mathrm{~Hz}$ oscillations lasting $0.5-5 \mathrm{~s}$ ) during non-REM sleep, which have been linked to deficits in sleepdependent memory consolidation and are associated with positive symptoms of schizophrenia and diminished thalamocortical connectivity [84]. It has been hypothesized that the decrease in spindle activity reflects impairment in the reticular thalamic neurons that are thought to be responsible for the generation of spindle activity and play a role in the process of gating whether sensory information is passed on to the cortex at the level of the thalamus [84].

There appears to be a complex causality relationship among schizophrenia and sleep problems. Sleep disturbance is quite common in the prodromal phase of schizophrenia and very often precedes psychotic exacerbations [83, 85, 86].

Schizophrenia is also associated with an increased rate of a number of sleep disorders. Although the prevalence of OSAin in antipsychotic-naive schizophrenic patients is unknown, a number of studies suggest that those patients taking antipsychotics have a significantly elevated rate of breathing-related sleep disorder (17-48\%) [87-92]. This is presumably a reflection of weight gain that occurs with these medications and possibly sedation or myorelaxant effects. Antipsychotic medications can also improve 
sleep disturbance, lead to daytime sedation, and can exacerbate RLS [39-41].

\section{SLEEP DISORDERS AND IMPACTS OF TREATMENTS ON PSYCHIATRIC CONDITIONS}

This section consists of a brief overview of a number of sleep disorders and outlines where treatments for these sleep disorders may have impacts on neuropsychiatric conditions. Treatments for sleep disorders could have effects on neuropsychiatric conditions in two ways. One is that therapies used to treat sleep disorders could be therapies for neuropsychiatric conditions in their own right or could directly exacerbate these conditions. The other is that treatments could improve sleep disorders and the improvement in those conditions could have a therapeutic effect on neuropsychiatric disorders. In this section, we review the available data on such relationships and consider indirect evidence for such effects.

\section{Insomnia}

Chronic insomnia disorder is defined as a report of experiencing difficulty falling asleep or staying asleep at least three times per week for at least 3 months occurring when there are adequate opportunity and circumstances for sleep and there are associated daytime consequences [93]. The prevalence of insomnia in the general population is estimated to be $\sim 10 \%$ [94, 95]. Primary treatments for this condition include cognitive behavioral therapy for insomnia as well as a number of different types of medications including benzodiazepines (e.g., triazolam, temazepam, clonazepam, etc.), the so-called "non-benzodiazepines" (zolpidem, zaleplon, eszopiclone, zopiclone), selective histamine-1 receptor antagonists, selective hypocretin/orexin receptor antagonists, melatonin receptor agonists, antidepressants (e.g., trazodone, mirtazapine, amitriptyline) and antipsychotics (e.g., lurasidone, quetiapine, olanzapine, risperidone), and over-the-counter agents (e.g., diphenhydramine, chlorpheniramine) [96-101].

There are some treatments administered for insomnia that have direct effects on neuropsychiatric disorders and could be used as therapies for those conditions. One example of this is mirtazapine. Although there are no double-blind, placebo-controlled trials of the effects of mirtazapine on insomnia, it is sometimes used to treat this condition clinically and there have been placebocontrolled studies of the $\mathrm{S}$ isomer of mirtazapine indicating insomnia efficacy [102]. When used to treat insomnia, mirtazapine is sometimes administered in dosages at or above $15 \mathrm{mg}$ where it has antidepressant effects established in double-blind, placebocontrolled trials. Another example is clonazepam, which is sometimes used clinically to treat insomnia and is an effective treatment for anxiety, including symptoms of GAD and panic disorder [103]. Further, a number of antipsychotic medications are sometimes used to treat insomnia and they all have been established to have effects on thought disorder and bipolar disorder in double-blind randomized, placebo-controlled trials, although the dosages used in those conditions are generally higher than the insomnia dosages [104]. Over-the-counter antihistamines, such as diphenhydramine, are commonly used for insomnia, and these agents have been found in epidemiologic studies to exacerbate the cognitive impairment of individuals with dementias due to the associated anti-cholinergic effects [105].

There is some evidence that the insomnia therapy eszopiclone may have direct antidepressant and anxiolytic effects. Studies were carried out evaluating the effects of eszopiclone therapy vs. placebo as co-therapy with open-label SSRI treatment in patients with insomnia co-morbid with MDD and GAD [10, 106, 107]. Eszopiclone not only significantly improved sleep compared with placebo but it also had a significant advantage over placebo on the associated MDD and GAD outcomes both with and without the sleep items included in the scales used. The initial interpretation of these findings was that eszopiclone improved sleep compared with placebo and that the sleep improvement mediated improvement in depression and anxiety. However, there is evidence that this is not the case. The identical studies were repeated with the insomnia agent zolpidem modifiedrelease and comparable improvement in sleep vs. placebo was observed but without an accompanying effect on depression or GAD symptoms $[108,109]$. The most parsimonious explanation for this set of findings is that eszopiclone has direct anxiolytic and antidepressant effects not mediated through effects on sleep and zolpidem modified-release does not.

In addition to having therapeutic effects on sleep onset and particularly sleep maintenance in insomnia patients, hypocretin/ orexin receptor antagonists have been of interest for potentially having direct therapeutic effects on a number of neuropsychiatric conditions, including major depression, anxiety disorders, and Alzheimer disease [110]. Support for the idea that hypocretin/ orexin antagonism might be therapeutic for major depression includes evidence that an animal model of stress-induced depression was associated with an increase in hypocretin/orexin neuronal signaling [111] and that a dual hypocretin/orexin antagonist had antidepressant-like effects in a rodent model of depression [112]. However, overall, studies carried out with animal models of depression and human studies of cerebrospinal fluid (CSF) hypocretin/orexin levels do not provide a clear indication that hypocretin/orexin antagonists have promise as antidepressants [110]. Consistent with this work, a pilot placebo-controlled, double-blind, randomized trial of the dual hypocretin/orexin antagonist filorexant did not indicate that this agent had significant antidepressant effects [113]. A potential role of hypocretin/orexin antagonists in the treatment of anxiety disorders is suggested by evidence that hypocretin/orexin neurons are activated by stress and, in turn, activate key systems thought to mediate anxiety, including norepinephrine and corticotrophin-releasing hormone [110]. Although no studies of hypocretin/orexin antagonists have yet been completed in humans that might provide a direct indication of therapeutic potential. Interest in using hypocretin-1/orexin-1 receptor-specific antagonists for those with post-traumatic stress syndrome has arisen based on an animal study, suggesting that such agents have promise as a means to facilitate the extinction of fear memories [114]. There is also interest in using hypocretin/orexin antagonists in those with Alzheimer disease driven by evidence that cognitive impairment, CSF $\tau$, and CSF A $\beta$ levels have been found to correlate with increased CSF orexin levels in people with this condition [115] and that dual hypocretin/orexin receptor antagonism decreased A-beta levels and plaque formation in amyloid precursor protein transgenic mice, a model of Alzheimer disease [116]. Lastly, the use of hypocretin/orexin antagonists for treatment of patients with substance use disorders has been spurred by reports that selective hypocretin-1/orexin-1 antagonism decreases ethanol consumption in ethanol-dependent mice [117] and hypocretin/orexin-2 antagonism decreases elevated heroin self-administration in rats [118].

In terms of the effects of improving sleep on neuropsychiatric conditions, two randomized studies have been carried out of the effects of cognitive behavior therapy for insomnia (CBT-I) vs. a behavioral control intervention on sleep and depression outcomes in patients with insomnia co-morbid with $\operatorname{MDD}[119,120]$. In both cases there was no difference in depression outcomes in the control and CBT-I groups, but statistically significant moderation of sleep on depression was seen in the CBT-I group. These studies suggest that it is not the case that improving sleep has a robust effect on improving depression; however, the mediation effects suggest the possibility of a small effect of improving sleep on depression outcome that is too small to manifest in a betweengroup difference.

There are a number of neuropsychiatric conditions where correlative effects have been observed, indicating that insomnia is 
170

linked to the course of the condition, but where data are lacking to determine if the relationship is causal such that treating insomnia might have a beneficial effect. This includes that sleep deprivation predisposes individuals to developing mania, although it has not been established if improving sleep in those with emerging mania prevents the development of episodes [38]. Insomnia is also a harbinger for episodes of exacerbation of psychosis in those with schizophrenia, but it has not been determined if treating insomnia mitigates these episodes [85, 86]. Insomnia increases risk of Alzheimer's disease but not all-cause dementia [121]. Insomnia also increases the risk of relapse to drinking in abstinent alcoholics $[67,68]$. In patients with TBI, sleep disturbance after the injury predicts the development of neuropsychological sequelae, including depression, anxiety, and apathy, at 6 and 12 months after injury and poor functional outcome at 1 year post trauma $[122,123]$. We lack data on whether treating these problems mitigates risk or slows onset/ progression, which would indicate a causal role.

There are a number of mechanistic implications of the above reviewed findings. The data indicating that eszopiclone may have antidepressant and anxiolytic effects is consistent with animal data indicating that the GABA-A receptor type to which agents bind has an impact on the clinical effects observed [124, 125]. The GABA-A receptor to which the benzodiazepines and nonbenzodiazepines bind is comprised of five peptides, one of which is referred to as the $a$-subunit $[124,125]$. The $a$-subunits that these agents bind to are of four types: $a-1, a-2, a-3$, and $a-5[124,125]$. Available animal data suggests that zolpidem modified-release, an agent lacking in anxiolytic or antidepressant effects, binds preferentially to a-1-containing GABA-A receptors, while eszopiclone, which has anxiolytic and antidepressant effects, and clonazepam, which has established anxiolytic effects, bind preferentially to GABA-A receptors containing $a-2$ and $a-3$ subunits $[126,127]$. These findings are consistent with a number of studies carried out in animals and suggest preferential binding to $a-2 / a-3$ containing GABA-A receptors as a mechanism of interest for antidepressant or anxiolytic therapies [124, 125]. Indeed, there have been efforts to develop such therapies and there is preliminary evidence of efficacy in humans [125].

The lack of compelling evidence indicating that treating insomnia improves depression outcomes has implications regarding the relationship of insomnia and depression. These data, when considered in light of the correlative epidemiologic evidence indicating that insomnia is a risk factor for future depression and predicts depression relapse, suggest that insomnia likely represents dysregulation that does not cause depression, but predicts depression and could reflect a role of chronic stress in the genesis of depression, which is supported by a substantial body of research $[128,129]$. Another possible explanation is that individuals prone to depression could be vulnerable to becoming dysregulated when stressed and, as a result, developing insomnia, which predicts future depression.

Obstructive sleep apnea

OSA is defined as repeated episodes occurring during sleep, where there is significant impediment to airflow in the upper airway, as manifested in events lasting at least $10 \mathrm{~s}$, where there is either a drop in airflow of $90 \%$ or greater, or a $>30 \%$ decrease in nasal pressure associated with at least a 3\% oxygen desaturation [130]. An estimated $25 \%$ of men and $10 \%$ of women will have OSA during their lifetime [131]. This number has been increasing rapidly, coincident with the rise in obesity in the population. If untreated, OSA is associated with daytime sleepiness and cognitive impairment and a host of cardiovascular and metabolic complications [132]. The primary treatment for this condition is continuous positive airway pressure (CPAP) therapy, which increases the pressure in the upper airway and maintains airway patency during sleep [133].
OSA has been reported to be associated with an adverse impact on a number of neuropsychiatric conditions. Like insomnia, it remains unresolved as to whether this is a correlative vs. causal connection in that we have limited data on whether the treatment of apnea ameliorates these adverse impacts.

One such association is that those with OSA have a greater rate of MDD than the general population [134]. A number of studies have been carried out assessing whether treatment for OSA with the standard therapy for this condition, CPAP, improves depression. A confound associated with this work is there is some overlap in symptoms among OSA and MDD such that improving daytime energy and nighttime sleep by treating OSA will appear as improvement in depression rating scales independent of whether MDD was present and improved. As such, some improvement in depression severity would be expected to occur with effective OSA therapy. Meta-analyses have been carried out attempting to determine if OSA treatment improves depression and, as expected, some improvement in depression has been observed, although significant effects of CPAP vs. sham therapy were not evident $[135,136]$. However, interpretation of these analyses must take into account that they evaluated studies that did not require subjects to have significant depression prior to initiation of treatment, which would be expected to decrease the likelihood of demonstrating significant effects on MDD. In this regard, one of the studies found that CPAP had a significant therapeutic effect vs. control treatment in studies where baseline depression severity was greatest but not in the rest of the studies carried out [136]. This evidence of efficacy must be viewed as quite preliminary because there were only two studies included in the group with more severe depression [135, 136].

OSA has also been found to be associated with an increased risk for developing all-cause dementia [121]. Several small studies provide preliminary evidence that CPAP may have a positive impact on those with dementia and OSA. One study found that 29 patients with mild cognitive impairment treated with CPAP experienced improved psychomotor/cognitive processing speed at 1 year compared to 25 who were non-adherent to CPAP [137]. In another study carried out in patients with Alzheimer's disease and OSA, 14 patients treated with CPAP experience a significantly slower median annual Mini-Mental State Examination decline than nine patients without CPAP [138]. In contrast, no difference in cognition was found between individuals who used CPAP vs. sham CPAP for 3 weeks who had OSA and Alzheimer's disease [139]. This difference in outcomes may reflect that CPAP treatment for more than 3 weeks is needed to achieve a beneficial effect on cognition in those with OSA and dementias. Taken together, these studies provide very preliminary evidence that sustained CPAP therapy might have a positive impact on cognition in those with OSA and dementia. It remains unclear whether CPAP might be improving a factor aggravating cognitive disturbances in those with dementia or whether CPAP could be addressing a factor that is fundamentally altering the course of dementias. However, preliminary evidence for a change in underlying dementia pathology is provided by one study, including 25 untreated patients with OSA and 10 OSA patients treated with CPAP, all of whom had "subjective cognitive impairment," which found that CPAP treatment was associated with lower CSF $\tau$ protein levels [140].

There is also evidence that CPAP therapy can have beneficial effects on PTSD symptoms in those with OSA and PTSD, although the mechanism for this remains unknown [141].

Lastly, very preliminary data suggest that CPAP could have a beneficial effect in those with OSA and schizophrenia [142]. The logic for this approach is simple and compelling. Many individuals with schizophrenia suffer from profound impairments in function; in fact, impairment in at least major area of function is required to diagnose this condition [14]. As a result, those with OSA and schizophrenia have to shoulder the added burden of daytime 
sleepiness and cognitive impairment that are nearly universally associated OSA on top of their already profound functional impairment. Any benefit that could be achieved by treating a reversible condition contributing to the impairments they experience could have significant impacts on the lives of affected individuals. This approach is illustrated by a pilot study carried out in only six individuals with OSA and schizophrenia, which revealed that CPAP improved the Brief Assessment of Cognition [142]. Hopefully, this pilot study will serve as the basis for identifying and treating OSA in those with schizophrenia and will inspire future research on the impact of CPAP on function in patients with schizophrenia.

\section{Parasomnias}

Parasomnias are a set of phenomena that occur during sleep in an episodic fashion and involve a behavior or experience not typical of sleep [93]. Examples include sleepwalking, sleep-related eating disorder, sleep terrors, and confusional arousals, nightmare disorder, and REM sleep behavior disorder [93]. A subset of these, sleepwalking, sleep-related eating disorder, sleep terrors, and confusional arousals, are believed to be partial arousals out of non-REM sleep, which typically is reflected in diminished responsiveness, impaired cognition, and amnesia for the event [93]. Others are believed to occur during REM sleep, including, nightmare disorder and REM sleep behavior disorder, where affected individuals act out their dreams because of an impairment in the paralysis that normally occurs during REM sleep [93]. The treatment with the best evidence base for improving these conditions is the benzodiazepine clonazepam [7].

Clonazepam not only has efficacy for the treatment of non-REM and REM parasomnias but it also has demonstrated efficacy in the treatment of some neuropsychiatric conditions, including seizure disorders and anxiety disorders. Clonazepam is a first-line treatment for acute seizures and has been found in double-blind, placebo-controlled trials to have efficacy for the treatment of several anxiety disorders, including GAD, panic disorder, and social anxiety disorder [143]. The therapeutic effects of clonazepam on both parasomnias and seizures may lead to the mis-impression that parasomnias are caused by seizure activity [144]. Although seizures, particularly those occurring in nocturnal frontal lobe epilepsy, can sometimes be confused with parasomnias, it is now well established that the classic non-REM and REM parasomnias (sleep-related eating disorder, sleepwalking, REM sleep behavior disorder, etc.) are not manifestations of seizures [144]. The mechanism of action of clonazepam on non-REM and REM parasomnias does not appear to be mediated by decreasing anxiety either as first-line therapies for anxiety disorders (SSRIs/ SNRIs) do not have robust therapeutic effects on non-REM or REM parasomnias [145].

Restless legs syndrome

RLS, also known as Willis-Ekbom disease, is a sleep-related movement disorder where affected individuals experience an urge to move their legs, often occurring with an uncomfortable sensory experience that is difficult to describe $[93,146]$. Symptoms are primarily experienced when at rest and there is usually some degree of relief of symptoms during movement [146]. Symptoms tend to be worst in the evening/night and interfere with the ability to fall asleep or return to sleep, but do not have any impact on sleep once sleep onset occurs [146]. The general population prevalence of RLS is around 3\% [147]. The primary treatments for this condition are dopamine receptor agonists such as pramipexole and ropinirole, L-Dopa, a precursor of dopamine, which increases dopamine release, and secondarily gabapentin and pregabalin, which are $a-2-\delta$ calcium channel blockers [146, 148].

Agents used to treat RLS also affect a number of neuropsychiatric conditions. Best established are the effects of dopamine agonists and L-Dopa for treating Parkinson disease (PD) [149]. That the same dopamine-related drugs have therapeutic effects in both conditions and that RLS is more common in those with PD than in the general population has led to speculation of a common underlying pathology [150]. Specifically some have hypothesized that RLS could be an early feature of PD [150]. Whether there are mechanistic links between RLS and PD remains unresolved; however, it is clear that RLS is independent of PD in that it is seen in association with many other conditions, including Tourette syndrome, Friedreich ataxia, polyneuropathy, iron deficiency anemia, and multiple sclerosis $[150,151]$.

There is preliminary evidence that dopamine agonists might have therapeutic effects in those with MDD. A statistically significant therapeutic effect of pramipexole was observed in a double-blind, randomized, placebo-controlled trial as add-on therapy to standard antidepressant medication in 60 patients with treatment-resistant MDD [152]. A therapeutic effect was also found in a study of 174 MDD patients randomized to pramipexole, fluoxetine, and placebo [153]. Lastly, a significant therapeutic effect was found in a study where 21 patients with bipolar depression were randomized to pramipexole or placebo given in addition to mood stabilizers [154]. A placebo-controlled trial of ropinirole was carried out in 231 patients with RLS and MDD and was found to have statistically significantly greater effects on depression and RLS compared with placebo [155]. This body of work provides some evidence that dopamine agonists might be expected to improve symptoms of MDD in those with RLS and suggests that these agents merit further study as potential MDD therapies.

Mechanistically, the therapeutic effects of dopamine agonists in MDD are consistent with studies in humans and animals, which provide some indication of dopamine system downregulation in individuals with this disorder [156]. This includes that downregulation of the dopamine transporter due to lower dopamine concentrations has been reported in patients with MDD [156]. Evidence of decreased dopamine turnover in MDD includes observations that patients with this condition have increased striatal D2 receptor binding [157] and that patients with MDD who committed suicide have been found to have elevated D2/3 receptor binding post mortem [158].

Another potential effect of dopamine agonists and L-Dopa on a neuropsychiatric disorder is that they might be expected to exacerbate schizophrenia based on psychotic symptoms being among the side effects of these agents [159]. Interestingly, there is some evidence that the opposite is the case for patients with schizophrenia treated with antipsychotic drugs, the reasons for which remain unclear. A meta-analysis of the effects of L-Dopa in antipsychotic-treated patients concluded that L-Dopa led to a statistically significant beneficial effect [160]. Otherwise, the only available data that addresses this issue is a pilot study in which pramipiexole or placebo was added to antipsychotic medication in 24 patients with schizophrenia or schizoaffective disorder, and pramipexole was found to have a significant therapeutic advantage over placebo with a modest rate of associated side effects [161]. The limited data available provides some reassurance when considering using L-Dopa or the dopamine agonists in patients with RLS and schizophrenia. In fact, some therapeutic effect on the schizophrenia might be expected.

\section{Circadian rhythm disorders}

Circadian rhythm disorders include a set of conditions where there is a disruption of sleep-wake rhythm, manifested in insomnia or excessive sleepiness, due to a misalignment between an individual's biological circadian rhythm and their desired or required sleep-wake schedule [93]. These conditions include delayed sleep-wake phase disorder; advanced sleep-wake phase disorder; irregular sleep-wake rhythm disorder; non-24-h sleep-wake rhythm disorder; and shift work disorder. The general 
population prevalence of advanced sleep-wake phase disorder, delayed sleep-wake phase disorder, and shift-work disorder are estimated to be $0.25-7.13 \%, 1.51-8.90 \%$, and $10-23 \%$, respectively $[162,163]$. Current primary therapy for these conditions includes attempting to shift an individual's endogenous rhythm through the use of targeted light exposure and melatonin administration or addressing symptoms as in the use of modafinil and $R$-modafinil for the treatment of shift-work sleep disorder [164].

Neuropsychiatric effects of the primary medication used to treat circadian rhythm disorders are of interest for mood disorders and those treated with antipsychotics. There has been interest for some time in the potential antidepressant effects of melatonin. The available research addressing this question suggests that there is no evidence indicating that melatonin had antidepressant effects; however, there are limitations to the available research and more research is needed to definitely determine if this is the case [165].

A number of studies have been carried out to determine if melatonin might have ameliorative effects on the metabolic side effects of atypical antipsychotics, which are commonly used to treat schizophrenia and bipolar disorder. A recent meta-analysis provides evidence that melatonin had beneficial effects on atypical antipsychotic-induced metabolic syndrome, which were greater in those with bipolar disorder than those with schizophrenia treated with these agents [166]. Given that many individuals with schizophrenia and bipolar disorder have circadian rhythm disorders, use of melatonin to address these problems in patients with these conditions treated with atypical antipsychotics seems particularly warranted.

\section{Future research directions}

This article has reviewed the impacts of treatments for sleep disorders on neuropsychiatric conditions and also considered how treatments for the neuropsychiatric conditions might affect sleep disorders. Clearly many treatments for sleep disorders affect neuropsychiatric conditions and many treatments for neuropsychiatric conditions affect sleep. This speaks of the strong interconnectedness of the two types of disorders. In some instances we have an understanding of the mechanisms relating sleep and neuropsychiatric conditions. However, in the vast majority cases we lack data on the nature of the inter-relatedness. This issue is most pressing with respect to addressing whether sleep problems that are associated with an increased risk of neuropsychiatric conditions are causal drivers of those neuropsychiatric conditions vs. having a non-causal association. The best way to answer this question is to carry out rigorous placebo-controlled trials assessing whether administering treatments for the sleep problem in question mitigates the risk for the development of the neuropsychiatric condition. The highest priorities for such studies are addressing whether: insomnia drives relapse in alcohol use disorder; insomnia plays a causal role in Alzheimer disease; insomnia plays a role in the development of symptoms post TBI; preventing sleep loss can prevent mania; inducing sleep can improve mania; treating insomnia can ward off episodes of acute psychosis in those with schizophrenia; and treating OSA improves depression, prevents or slows onset of dementias, and improves function in those with schizophrenia. Further, there is work needed to determine the mechanisms underlying any causal relationships found and for why a number of treatments for sleep disorders have beneficial effects for neuropsychiatric conditions. Examples are the beneficial effects of clonazepam on both parasomnias and seizure disorders/anxiety, the therapeutic effects of L-Dopa and dopamine agonists on both RLS and depression and possibly schizophrenia, and the effects of melatonin on circadian rhythm disorders and atypical antipsychotic-associated metabolic syndrome. Lastly, it is hoped that bringing the cross-therapeutic effects of agents used to treat sleep disorders and neuropsychiatric disorders will serve as a guide to clinicians to help in the optimization of care for their patients with these conditions.

\section{FUNDING AND DISCLOSURE}

Dr. Krystal received no funding for this work. He has conflicts of interest based on the following grant funding and consultant relationships: Grants/research support: NIH, Janssen, Jazz. Axsome, Reveal Biosensors. Consultant: Adare, Eisai, Ferring, Galderma, Harmony Biosciences, Idorsia, Jazz, Janssen, Takeda, Merck, Neurocrine, Pernix, Physician's Seal.

\section{ADDITIONAL INFORMATION}

Publisher's note: Springer Nature remains neutral with regard to jurisdictional claims in published maps and institutional affiliations.

\section{REFERENCES}

1. Winsky-Sommerer R, de Oliveira P, Loomis S, Wafford K, Dijk DJ, Gilmour G. Disturbances of sleep quality, timing and structure and their relationship with other neuropsychiatric symptoms in Alzheimer's disease and schizophrenia: insights from studies in patient populations and animal models. Neurosci Biobehav Rev. 2019;97:112-37.

2. Sateia MJ. Update on sleep and psychiatric disorders. Chest. 2009;135:1370-9.

3. Nadorff MR, Drapeau CW, Pigeon WR. Psychiatric illness and sleep in older adults: comorbidity and opportunities for intervention. Sleep Med Clin. 2018;13:81-91.

4. Stubbs B, Vancampfort D, Veronese N, Solmi M, Gaughran F, Manu P, et al. The prevalence and predictors of obstructive sleep apnea in major depressive disorder, bipolar disorder and schizophrenia: a systematic review and metaanalysis. J Affect Disord. 2016;197:259-67.

5. Heck T, Zolezzi M. Obstructive sleep apnea: management considerations in psychiatric patients. Neuropsychiatr Dis Treat. 2015;11:2691-8.

6. Sutton EL. Psychiatric disorders and sleep issues. Med Clin N Am. 2014;98:1123-43.

7. Proserpio $P$, Terzaghi M, Manni R, Nobili L. Drugs used in parasomnia. Sleep Med Clin. 2018;13:191-202.

8. Perna G, Alciati A, Riva A, Micieli W, Caldirola D. Long-term pharmacological treatments of anxiety disorders: an updated systematic review. Curr Psychiatry Rep. 2016;18:23

9. Murphy MJ, Peterson MJ. Sleep disturbances in depression. Sleep Med Clin. 2015;10:17-23.

10. Fava M, McCall WV, Krystal A, Rubens R, Caron J, Wessel T, et al. Eszopiclone coadministered with fluoxetine in patents with insomnia co-existing with major depressive disorder. Biol Psychiatry. 2006;59:1052-60.

11. Krystal AD, Zammit G. The sleep effects of lurasidone: a placebo-controlled cross-over study using a 4-hour phase-advance model of transient insomnia. Hum Psychopharmacol. 2016;31:206-16.

12. Krystal AD, Thase ME, Tucker VL, Goodale EP. Bupropion HCL and sleep in patients with depression. Curr Psychiatry Rev. 2007;3:123-8.

13. Krystal AD. Antidepressant and Antipsychotic drugs. Sleep Med Clin. 2010;5:571-89.

14. American Psychiatric Association. Diagnostic and statistical manual of mental disorders. 5th ed. Washington: American Psychiatric Association; 2013.

15. Kessler RC, Bromet EJ. The epidemiology of depression across cultures. Annu Rev Public Health. 2013;34:119-38.

16. Kessler RC, Berglund P, Demler O, et al. The epidemiology of major depressive disorder: results from the National Comorbidity Survey Replication (NCS-R). J Am Med Assoc. 2003;289:3095-105.

17. Friedrich MJ. Depression is the leading cause of disability around the world. J Am Med Assoc. 2017;317:1517.

18. Yates WR, Mitchell J, Rush AJ, et al. Clinical features of depressed outpatients with and without co-occurring general medical conditions in STAR* D. Gen Hosp Psychiatry. 2004;26:421-9.

19. Liu X, Buysse DJ, Gentzler AL, et al. Insomnia and hypersomnia associated with depressive phenomenology and comorbidity in childhood depression. Sleep. 2007;30:83-90

20. Benca RM, Obermeyer WH, Thisted RA, Gillin JC. Sleep and psychiatric disorders: a meta-analysis. Arch Gen Psychiatry. 1992;49:651-68. 
21. Arfken C, Joseph A, Sandhu G, et al. The status of sleep abnormalities as a diagnostic test for major depressive disorder. J Affect Disord. 2014;156:36-45.

22. Borbely AA, Tobler I, Loepfe M, et al. All-night spectral analysis of the sleep EEG in untreated depressives and normal controls. Psychiatry Res. 1984;12:27-33.

23. Kupfer DJ, Reynolds CF III, Ulrich RF, Grochocinski VJ. Comparison of automated REM and slow-wave sleep analysis in young and middle-aged depressed subjects. Biol Psychiatry. 1986;21:189-200.

24. Johnson EO, Roth T, Breslau N. The association of insomnia with anxiety disorders and depression: exploration of the direction of risk. J Psychiatr Res. 2006;40:700-8.

25. Franzen PL, Buysse DJ. Sleep disturbances and depression: risk relationships for subsequent depression and therapeutic implications. Dialog Clin Neurosci. 2008;10:473-81.

26. Stubbs B, Vancampfort D, Veronese N, Solmi M, Gaughran F, Manu P, et al. The prevalence and predictors of obstructive sleep apnea in major depressive disorder, bipolar disorder and schizophrenia: a systematic review and metaanalysis. J Affect Disord. 2016;197:259-67.

27. Hoque R, Chesson AL, Jr. Pharmacologically induced/exacerbated restless legs syndrome, periodic limb movements of sleep, and REM behavior disorder/REM sleep without atonia: literature review, qualitative scoring, and comparative analysis. J Clin Sleep Med. 2010;6:79-83.

28. Yang C, White DP, Winkelman JW. Antidepressants and periodic leg movements of sleep. Biol Psychiatry. 2005;58:510-4.

29. Kolla BP, Mansukhani MP, Bostwick JM. The influence of antidepressants on restless legs syndrome and periodic limb movements: a systematic review. Sleep Med Rev. 2018;38:131-40.

30. McCarter SJ, St Louis EK, Sandness DJ, Arndt K, Erickson M, Tabatabai G, et al. Antidepressants increase REM sleep muscle tone in patients with and without REM sleep behavior disorder. Sleep. 2015;38:907-17.

31. Winkelman JW, James L. Serotonergic antidepressants are associated with REM sleep without atonia. Sleep. 2004;27:317-21.

32. Merikangas KR, Akiskal HS, Angst J, Greenberg PE, Hirschfeld RM, Petukhova M et al. Lifetime and 12-month prevalence of bipolar spectrum disorder in the National Comorbidity Survey replication. Arch Gen Psychiatry. 2007;64:543-52.

33. Nofzinger EA, Thase ME, Reynolds CF,III. et al. Hypersomnia in bipolar depression: a comparison with narcolepsy using the multiple sleep latency test. Am J Psychiatry. 1991;148:1177-81.

34. Harvey AG, Schmidt DA, Scarna A, et al. Sleep-related functioning in euthymic patients with bipolar disorder, patients with insomnia, and subjects without sleep problems. Am J Psychiatry. 2005;162:50-7.

35. Giles DE, Rush AJ, Roffwarg HP. Sleep parameters in bipolar I, bipolar II, and unipolar depressions. Biol Psychiatry. 1986;21:1340-3.

36. Hudson Jl, Lipinski JF, Frankenburg FR, et al. Electroencephalographic sleep in mania. Arch Gen Psychiatry. 1988;45:267-73.

37. Ritter PS, Marx C, Bauer M, et al. The role of disturbed sleep in the early recognition of bipolar disorder: a systematic review. Bipolar Disord. 2011;13:227-37

38. Wehr TA, Goodwin FK, Wirz-Justice A, et al. 48-Hour sleep-wake cycles in manicdepressive illness: naturalistic observations and sleep deprivation experiments. Arch Gen Psychiatry. 1982;39:559-65.

39. Krystal AD. Antidepressant and antipsychotic drugs. Sleep Med Clin. 2010;5:571-89.

40. Krystal AD, Goforth HW, Roth T. Effects of antipsychotic medications on sleep in schizophrenia. Int Clin Psychopharmacol. 2008;23:150-60.

41. Patatanian E, Claborn MK. Drug-induced restless legs syndrome. Ann Pharmacother. 2018;52:662-72.

42. Kessler RC, Petukhova M, Sampson NA, et al. Twelve-month and lifetime prevalence and lifetime morbid risk of anxiety and mood disorders in the US. Int J Methods Psychiatr Res. 2012;21:169-84.

43. Seedat S, Scott KM, Angermeyer MC, et al. Cross-national associations between gender and mental disorders in the World Health Organization World Mental Health Surveys. Arch Gen Psychiatry. 2009;66:785-95.

44. Monti JM, Monti D. Sleep disturbance in generalized anxiety disorder and its treatment. Sleep Med Rev. 2000;4:263-76.

45. DeMartini J, Patel G, Fancher TL. Generalized anxiety disorder. Ann Intern Med. 2019:170:ITC49-ITC64.

46. Kessler RC, Berglund P, Demler O, et al. Lifetime prevalence and age-of-onset distributions of DSM-IV disorders in the National Comorbidity Survey Replication. Arch Gen Psychiatry. 2005;62:593-602.

47. Ohayon MM, Shapiro CM. Sleep disturbances and psychiatric disorders associated with posttraumatic stress disorder in the general population. Compr Psychiatry. 2000;41:469-78.

48. McLay RN, Klam WP, Volkert SL. Insomnia is the most commonly reported symptom and predicts other symptoms of post-traumatic stress disorder in U.S. service members returning from military deployments. Mil Med. 2010;175:759-62.

49. Pruiksma KE, Taylor DJ, Ruggero $C$, et al. A psychometric study of the fear of sleep inventory-short form (FoSI-SF). J Clin Sleep Med. 2014;10:551-8.

50. Mellman TA, Bustamante V, Fins Al, Pigeon WR, Nolan B. REM sleep and the early development of posttraumatic stress disorder. Am J Psychiatry. 2002;159:1696-701.

51. Breslau N, Roth T, Burduvali E, Kapke A, Schultz L, Roehrs T. Sleep in lifetime posttraumatic stress disorder: a community-based polysomnographic study. Arch Gen Psychiatry. 2004;61:508-16.

52. Gehrman P, Seelig AD, Jacobson IG, et al. Predeployment sleep duration and insomnia symptoms as risk factors for new-onset mental health disorders following military deployment. Sleep. 2013;36:1009-18.

53. Mellman TA, Pigeon WR, Nowell PD, Nolan B. Relationships between REM sleep findings and PTSD symptoms during the early aftermath of trauma. J Trauma Stress. 2007;20:893-901.

54. Macera CA, Aralis HJ, Rauh MJ, et al. Do sleep problems mediate the relationship between traumatic brain injury and development of mental health symptoms after deployment?. Sleep. 2013;36:83-90.

55. Fehr BS, Katz WF, Van Enkevort EA, Khawaja IS. Obstructive sleep apnea in posttraumatic stress disorder comorbid with mood disorder: significantly higher incidence than in either diagnosis alone. Prim Care Companion CNS Disord. 2018;20. pii: 18m02281. https://doi.org/10.4088/PCC.18m02281.

56. Mysliwiec V, Brock MS, Creamer JL, O'Reilly BM, Germain A, Roth BJ. Trauma associated sleep disorder: a parasomnia induced by trauma. Sleep Med Rev. 2018;37:94-104.

57. Hoskins M, Pearce J, Bethell A, Dankova L, Barbui C, Tol WA, et al. Pharmacotherapy for post-traumatic stress disorder: systematic review and metaanalysis. Br J Psychiatry. 2015;206:93-100.

58. Raskind MA, Peskind ER, Chow B, Harris C, Davis-Karim A, Holmes HA, et al. Trial of prazosin for post-traumatic stress disorder in military veterans. N Engl J Med. 2018;378:507-17.

59. Taylor FB, Martin P, Thompson C, Williams J, Mellman TA, Gross C, et al. Prazosin effects on objective sleep measures and clinical symptoms in civilian trauma posttraumatic stress disorder: a placebo-controlled study. Biol Psychiatry. 2008;63:629-32.

60. Pleis JR, Ward BW, Lucas JW. Summary health statistics for U.S. adults: National Health Interview Survey, 2009.National Center for Health Statistics. Vital Health Stat. 2010;10:1-207.

61. Papineau K, Roehrs T, Petrucelli N, et al. Electrophysiological assessment (The Multiple Sleep Latency Test) of the biphasic effects of ethanol in humans. Alcohol Clin Exp Res. 1998;22:231-5.

62. Stone BM. Sleep and low doses of alcohol. Electro Clin Neurophysiol. 1980;48:706-9.

63. Roehrs T, Roth T. Sleep, sleepiness, sleep disorders and alcohol use and abuse. Sleep Med Rev. 2001;5:287-97.

64. Roehrs T, Roth T. Medication and substance abuse. In: Kryger M, Roth T, Dement WC, editors. Principles and practice of sleep medicine. 5th ed. Part II, Section 16. St. Louis: Elsevier Saunders; 2010. p. 1512-23.

65. Brower KJ. Alcohol's effects on sleep in alcoholics. Alcohol Res Health. 2001;25:110-25.

66. Drummond SPA, Gillin JC, Smith TL, Demondena A. The sleep of abstinent pure primary alcoholic patients: natural course and relation to relapse. Alcohol Clin Exp Res. 1998;22:1796-802.

67. Allen RP, Wagman AM, Funderburk FR, Well DT. Slow wave sleep: a predictor of individuals differences in response to drinking? Biol Psychiatry. 1980;15:345-8.

68. Gillin JC, Smith TL, Irwin M, et al. Increased pressure for rapid eye movement sleep at time of hospital admission predicts relapse in nondepressed patients with primary alcoholism at 3-month follow-up. Arch Gen Psychiatry. 1994;51:189-97.

69. Gillin JC, Drummond SPA. Medication and substance abuse. In: Kryger MH, Roth T, Dement WC, editors. Principles and practice of sleep medicine. 3rd ed. Philadelphia: Saunders; 2000. p. 1176-95.

70. Issa FG, Sullivan CE. Alcohol, snoring and sleep apneas. J Neurol Neurosurg Psychiatry. 1982;45:353-9.

71. Mitler MM, Dawson A, Henriksen SJ, et al. Bedtime ethanol increases resistance of upper airways and produces sleep apneas in asymptomatic snorers. Alcohol Clin Exp Res. 1988;12:801-5.

72. McGrath J, Saha S, Chant D, et al. Schizophrenia: a concise overview of incidence, prevalence, and mortality. Epidemiol Rev. 2008;30:67-76.

73. Klingaman EA, Palmer-Bacon J, Bennett ME, Rowland LM. Sleep disorders among people with schizophrenia: emerging research. Curr Psychiatry Rep. 2015;17:79

74. Anderson KN, Bradley AJ. Sleep disturbance in mental health problems and neurodegenerative disease. Nat Sci Sleep. 2013;5:61-75. 
75. Monti JM, et al. Sleep and circadian rhythm dysregulation in schizophrenia. Prog Neuropsychopharmacol Biol Psychiatry. 2013;43:209-16.

76. Hofstetter JR, Mayeda AR, Happel CG, et al. Sleep and daily activity preferences in schizophrenia: associations with neurocognition and symptoms. J Nerv Ment Dis. 2003;191:408-10.

77. Chouinard S, Poulin J, Stip E, et al. Sleep in untreated patients with schizophrenia: a meta-analysis. Schizophr Bull. 2004;30:957-67.

78. Sarkar S, Katshu M, Nizamie SH, et al. Slow wave sleep deficits as a trait marker in patients with schizophrenia. Schizophr Res. 2010;124:127-33.

79. Poulin J, Daoust A, Forest $\mathrm{G}$, et al. Sleep architecture and its clinical correlates in first episode and neuroleptic-naive patients with schizophrenia. Schizophr Res. 2003;62:147-53.

80. Van Kammen DP, Van Kammen WM, Peters J, et al. Decreased slow-wave sleep and enlarged lateral ventricles in schizophrenia. Neuropsychopharmacology. 1988;1:265-71.

81. Keshavan MS, Miewald J, Haas G, et al. Slow-wave sleep and symptomatology in schizophrenia and related psychotic disorders. J Psychiatr Res. 1995; 29:303-14.

82. Tandon R, Shipley JE, Taylor S, et al. Electroencephalographic sleep abnormalities in schizophrenia. Relationship to positive/negative symptoms and prior neuroleptic treatment. Arch Gen Psychiatry. 1992;49:185-94.

83. Lauer CJ, Schreiber W, Pollmacher T, et al. Sleep in schizophrenia: a polysomnographic study o drug-naive patients. Neuropsychopharmacology. 1997;16:1-60.

84. Manoach DS, Stickgold R. Abnormal sleep spindles, memory consolidation, and schizophrenia. Annu Rev Clin Psychol. 2019;15:451-79.

85. Zanini $M$, et al. Do sleep abnormalities and misaligned sleep/circadian rhythm patterns represent early clinical characteristics for developing psychosis in high risk populations? Neurosci Biobehav Rev. 2013;37(10, Part 2):2631-7.

86. Benson KL. Sleep in schizophrenia. Sleep Med Clin. 2008;3:251-60.

87. Shirani A, Paradiso S, Dyken ME. The impact of atypical antipsychotic use on obstructive sleep apnea: a pilot study and literature review. Sleep Med. 2011;12:591-7.

88. Lin WC, Winkelman JW. Obstructive sleep apnea and severe mental illness: evolution and consequences. Curr Psychiatry Rep. 2012;14:503-10.

89. Ancoli-lsrael S, Martin J, Jones DW, et al. Sleep disordered breathing and periodic limb movements in sleep in older patients with schizophrenia. Biol Psychiatry. 1999;45:1426-32.

90. Benson KL, Zarcone VP. Sleep abnormalities in schizophrenia and other psychotic disorders. Rev Psychiatry. 1994;13:677-705.

91. Takahashi Kl, Shimizu T, Sugita T, et al. Prevalence of sleep-related respiratory disorders in 101 schizophrenic patients. Psychiatry Clin Neurosci. 1998;52:229-31.

92. Waters F, Hanken K, Rock D. Sleep-disordered breathing in schizophrenia: an audit. Schiz Res. 2013;143:393-4.

93. American Academy of Sleep Medicine. International classification of sleep disorders. 3rd ed. Darien: American Academy of Sleep Medicine; 2014

94. Ohayon MM. Epidemiology of insomnia: what we know and what we still need to learn. Sleep Med Rev. 2002;6:97-111.

95. Ohayon MM. Observation of the natural evolution of insomnia in the American general population cohort. Sleep Med Clin. 2009;4:87-92.

96. Sateia MJ, Buysse DJ, Krystal AD, Neubauer DN, Heald JL. Clinical practice guideline for the pharmacologic treatment of chronic insomnia in adults: an american academy of sleep medicine clinical practice guideline. J Clin Sleep Med. 2017;13:307-49.

97. Krystal AD. Current, emerging, and newly available insomnia medications. J Clin Psychiatry. 2015;76:e1045. PMID: 26335094

98. Minkel J, Krystal AD. Optimizing the pharmacologic treatment of insomnia: current status and future horizons. Sleep Med Clin. 2013;8:333-50.

99. Richey SM, Krystal AD. Pharmacological advances in the treatment of insomnia. Curr Pharm Des. 2011:17:1471-5.

100. Krystal AD. A compendium of placebo-controlled trials of the risks/benefits of pharmacological treatments for insomnia: the empirical basis for US clinical practice. Sleep Med Rev. 2009;13:265-74.

101. Krystal AD, Edinger JD. Sleep EEG predictors and correlates of the response to cognitive behavioral therapy for insomnia. Sleep. 2010;33:669-77.

102. Ivgy-May N, Ruwe F, Krystal A, Roth T. Esmirtazapine in non-elderly adult patients with primary insomnia: efficacy and safety from a randomized, 6-week sleep laboratory trial. Sleep Med. 2015;16:838-44.

103. Nardi AE, Perna G. Clonazepam in the treatment of psychiatric disorders: an update. Int Clin Psychopharmacol. 2006;21:131-42.

104. Thompson W, Quay TAW, Rojas-Fernandez C, Farrell B, Bjerre LM. Atypical antipsychotics for insomnia: a systematic review. Sleep Med 2016;22:13-17.

105. Krystal AD, Richelson $E$, Roth $T$. Review of the histamine system and the clinical effects of $\mathrm{H} 1$ antagonists: basis for a new model for understanding the effects of insomnia medications. Sleep Med Rev. 2013;17:263-72.
106. Krystal AD, Fava M, Rubens $R$, Wesel $T$, Caron J, Wilson $P$, et al. Evaluation of eszopiclone discontinuation after co-therapy with fluoxetine for insomnia with co-existing depression. J Clin Sleep Med. 2007;3:48-55.

107. Pollack M, Kinrys G, Krystal A, McCall WV, Roth T, Schaefer K, et al. Eszopiclone coadministered with escitalopram in patients with insomnia and comorbid generalized anxiety disorder. Arch Gen Psychiatry. 2008;65:551-62.

108. Fava M, Asnis GM, Shrivastava RK, Lydiard B, Bastani B, Sheehan DV, et al. Improved insomnia symptoms and sleep-related next-day functioning in patients with comorbid major depressive dis-order and insomnia following concomitant zolpidem extended-release $12.5 \mathrm{mg}$ and escitalopram treatment: a randomized controlled trial. J Clin Psychiatry. 2011;72:914-28.

109. Fava M, Asnis GM, Shrivastava R, Lydiard B, Bastani B, Sheehan D, et al. Zolpidem extended-release improves sleep and next-day symptoms in comorbid insomnia and generalized anxiety disorder. J Clin Psychopharmacol. 2009;29: 222-30.

110. Herring WJ, Roth T, Krystal AD, Michelson D. Orexin receptor antagonists for the treatment of insomnia and potential treatment of other neuropsychiatric indications. J Sleep Res. 2019;28:e12782.

111. Jalewa J, Wong-Lin K, McGinnity TM, Prasad G, Hölscher C. Increased number of orexin/hypocretin neurons with high and prolonged external stress-induced depression. Behav Brain Res. 2014;272:196-204.

112. Nollet M, Gaillard P, Tanti A, Girault V, Belzung C, Leman S. Neurogenesisindependent antidepressant-like effects on behavior and stress axis response of a dual orexin receptor antagonist in a rodent model of depression. Neuropsychopharmacology. 2012;37:2210-21.

113. Connor KM, Ceesay $P$, Hutzelmann J, Snavely D, Krystal AD, Trivedi MH, et al. Phase II proof-of-concept trial of the orexin receptor antagonist filorexant (MK6096) in patients with major depressive disorder. Int J Neuropsychopharmacol. 2017;20:613-8.

114. Flores Á, Valls-Comamala V, Costa G, Saravia R, Maldonado R, Berrendero F. The hypocretin/orexin system mediates the extinction of fear memories. Neuropsychopharmacology. 2014;39:2732-41.

115. Liguori C, Romigi A, Nuccetelli M, Zannino S, Sancesario G, Martorana A, et al. Orexinergic system dysregulation, sleep impairment, and cognitive decline in Alzheimer disease. JAMA Neurol. 2014;71:1498-505.

116. Kang JE, Lim MM, Bateman RJ, Lee JJ, Smyth LP, Cirrito JR, et al. Amyloid-beta dynamics are regulated by orexin and the sleep-wake cycle. Science. 2009;326:1005-7.

117. Lopez MF, Moorman DE, Aston-Jones G, Becker HC. The highly selective orexin/ hypocretin-1 receptor antagonist GSK1059865 potently reduces ethanol drinking in ethanol dependent mice. Brain Res. 2016;1636:74-80.

118. Schmeichel BE, Barbier E, Misra KK, Contet C, Schlosburg JE, Grigoriadis D, et al. Hypocretin receptor 2 antagonism dose-dependently reduces escalated heroin self-administration in rats. Neuropsychopharmacology. 2015;40:1123-9.

119. Manber R, Buysse DJ, Edinger J, Krystal A, Luther JF, Wisniewski SR, et al. Efficacy of cognitive-behavioral therapy for insomnia combined with antidepressant pharmacotherapy in patients with comorbid depression and insomnia: a randomized controlled trial. J Clin Psychiatry. 2016;77:e1316-23.

120. Carney CE, Edinger JD, Kuchibhatla M, Lachowski AM, Bogouslavsky O, Krystal $A D$, et al. Cognitive behavioral insomnia therapy for those with insomnia and depression: a randomized controlled clinical trial. Sleep. 2017:40. https://doi.org/ 10.1093/sleep/zsx019.

121. Shi L, Chen SJ, Ma MY, Bao YP, Han Y, Wang YM, et al. Sleep disturbances increase the risk of dementia: a systematic review and meta-analysis. Sleep Med Rev. 2018;40:4-16.

122. Baumann $C R$, Werth $E$, Stocker $R$, Ludwig $S$, Bassetti CL. Sleep-wake disturbances 6 months after traumatic brain injury: a prospective study. Brain . 2007;130(Part 7):1873-83.

123. Sandsmark DK, Elliott JE, Lim MM. Sleep-wake disturbances after traumatic brain injury: synthesis of human and animal studies. Sleep. 2017;40. https://doi. org/10.1093/sleep/zsx044.

124. Sieghart W. Allosteric modulation of GABAA receptors via multiple drug-binding sites. Adv Pharm. 2015;72:53-96.

125. Chen X, van Gerven J, Cohen A, Jacobs G. Human pharmacology of positive GABA-A subtype-selective receptor modulators for the treatment of anxiety. Acta Pharmacol Sin. 2018.40:571-82.

126. Nutt DJ, Stahl SM. Searching for perfect sleep: the continuing evolution of GABAA receptor modulators as hypnotics. J Psychopharmacol. 2010;24:1601-12.

127. Jia $F$, Goldstein PA, Harrison NL. The modulation of synaptic GABA(A) receptors in the thalamus by eszopiclone and zolpidem. J Pharm Exp Ther. 2009;328:1000-6.

128. Lee EH, Han PL. Reciprocal interactions across and within multiple levels of monoamine and cortico-limbic systems in stress-induced depression: a systematic review. Neurosci Biobehav Rev. 2019;101:13-31. 
129. van Praag HM. Can stress cause depression? Prog Neuropsychopharmacol Biol Psychiatry. 2004;28:891-907.

130. Berry RB, Brooks R, Gamaldo CE, et al. for the American Academy of Sleep Medicine. The AASM manual for the scoring of sleep and associated events: rules, terminology and technical specifications. Version 2.4. Darien: American Academy of Sleep Medicine; 2017.

131. Young T, Palta M, Dempsey J, Peppard PE, Nieto FJ, Hla KM. Burden of sleep apnea: rationale, design, and major findings of the Wisconsin Sleep Cohort study. WMJ. 2009;108:246-9.

132. Punjabi NM. The epidemiology of adult obstructive sleep apnea. Proc Am Thorac Soc. 2008;5:136-43.

133. Lévy $P$, Kohler $M$, McNicholas WT, Barbé F, McEvoy RD, Somers VK, et al. Obstructive sleep apnoea syndrome. Nat Rev Dis Prim. 2015;1:15015.

134. BaHammam AS, Kendzerska T, Gupta R, Ramasubramanian C, Neubauer DN, Narasimhan $\mathrm{M}$, et al. Comorbid depression in obstructive sleep apnea: an underrecognized association. Sleep Breath. 2016;20:447-56.

135. Gupta MA, Simpson FC, Lyons DC. The effect of treating obstructive sleep apnea with positive airway pressure on depression and other subjective symptoms: a systematic review and meta-analysis. Sleep Med Rev. 2016;28:55-68.

136. Povitz M, Bolo CE, Heitman SJ, Tsai WH, Wang J, James MT. Effect of treatment of obstructive sleep apnea on depressive symptoms: systematic review and metaanalysis. PLoS Med. 2014;11:e1001762.

137. Richards KC, Gooneratne N, Dicicco B, Hanlon A, Moelter S, Onen F, et al. CPAP adherence may slow 1-year cognitive decline in older adults with mild cognitive impairment and apnea. J Am Geriatr Soc. 2019;67:558-64.

138. Troussière $A C$, Charley $C M$, Salleron J, Richard F, Delbeuck $X$, Derambure $P$, et al Treatment of sleep apnoea syndrome decreases cognitive decline in patients with Alzheimer's disease. J Neurol Neurosurg Psychiatry. 2014;85:1405-8.

139. Ancoli-Israel S, Palmer BW, Cooke JR, Corey-Bloom J, Fiorentino L, Natarajan L, et al. Cognitive effects of treating obstructive sleep apnea in Alzheimer's disease: a randomized controlled study. J Am Geriatr Soc. 2008;56:2076-81.

140. Liguori C, Mercuri NB, Izzi F, Romigi A, Cordella A, Sancesario G, et al. Obstructive sleep apnea is associated with early but possibly modifiable Alzheimer's disease biomarkers changes. Sleep. 2017;40. https://doi.org/10.1093/sleep/zsx011.

141. Zhang Y, Ren R, Yang L, Zhou J, Sanford LD, Tang X. The effect of treating obstructive sleep apnea with continuous positive airway pressure on posttraumatic stress disorder: A systematic review and meta-analysis with hypothetical model. Neurosci Biobehav Rev. 2019;102:172-83.

142. Myles H, Myles N, Coetzer CLC, Adams R, Chandratilleke M, Liu D, et al. Cognition in schizophrenia improves with treatment of severe obstructive sleep apnoea: a pilot study. Schizophr Res Cogn. 2018;15:14-20.

143. Wang SM, Kim JB, Sakong JK, Suh HS, Oh KS, Woo JM, et al. The efficacy and safety of clonazepam in patients with anxiety disorder taking newer antidepressants: a multicenter naturalistic study. Clin Psychopharmacol Neurosci. 2016;14:177-83.

144. Tinuper $P$, Bisulli $F$, Provini $F$. The parasomnias: mechanisms and treatment. Epilepsia. 2012;53 Suppl. 7:12-9.

145. Kierlin L, Littner MR. Parasomnias and antidepressant therapy: a review of the literature. Front Psychiatry. 2011;2:71

146. Iranzo A. Parasomnias and sleep-related movement disorders in older adults. Sleep Med Clin. 2018;13:51-61.

147. Allen RP, Walters AS, Montplaisir J, Hening W, Myers A, Bell TJ, et al. Restless legs syndrome prevalence and impact: REST general population study. Arch Intern Med. 2005;165:1286-92.

148. Aurora RN, Kristo DA, Bista SR, Rowley JA, Zak RS, Casey KR. American Academy of Sleep Medicine, et al. The treatment of restless legs syndrome and periodic limb movement disorder in adults—an update for 2012: practice parameters with an evidence-based systematic review and meta-analyses: an American Academy of Sleep Medicine Clinical Practice Guideline. Sleep. 2012;35:1039-62.

149. Reich SG, Savitt JM. Parkinson's disease. Med Clin N Am. 2019;103:337-50.

150. Ferini-Strambi L, Carli G, Casoni F, Galbiati A. Restless legs syndrome and parkinson disease: a causal relationship between the two disorders? Front Neurol. 2018;9:551.

151. Alonso-Navarro H, García-Martín E, Agúndez JAG, Jiménez-Jiménez FJ. Association between restless legs syndrome and other movement disorders. Neurology. 2019;92:948-64. https://doi.org/10.1212/WNL.0000000000007500.

152. Cusin C, lovieno N, losifescu DV, Nierenberg AA, Fava M, Rush AJ, et al. A randomized, double-blind, placebo-controlled trial of pramipexole augmentation in treatment-resistant major depressive disorder. J Clin Psychiatry. 2013;74: e636-41.

153. Corrigan $\mathrm{MH}$, Denahan $\mathrm{AQ}$, Wright $\mathrm{CE}$, Ragual RJ, Evans DL. Comparison of pramipexole, fluoxetine, and placebo in patients with major depression. Depress Anxiety. 2000;11:58-65.

154. Zarate CA Jr., Payne JL, Singh J, Quiroz JA, Luckenbaugh DA, Denicoff KD, et al. Pramipexole for bipolar II depression: a placebo-controlled proof of concept study. Biol Psychiatry. 2004;56:54-60.

155. Benes H, Mattern W, Peglau I, Dreykluft T, Bergmann L, Hansen C, et al. Ropinirole improves depressive symptoms and restless legs syndrome severity in RLS patients: a multicentre, randomized, placebo-controlled study. J Neurol. 2011;258:1046-54.

156. Belujon P, Grace AA. Dopamine system dysregulation in major depressive disorders. Int J Neuropsychopharmacol. 2017;20:1036-46.

157. D'Haenen HA, Bossuyt A. Dopamine D2 receptors in depression measured with single photon emission computed tomography. Biol Psychiatry. 1995;35:128-32.

158. Pare CM, Yeung DP, Price K, Stacey RS. 5-Hydroxytryptamine, noradrenaline, and dopamine in brainstem, hypothalamus, and caudate nucleus of controls and of patients committing suicide by coal-gas poisoning. Lancet. 1969;2:133-5.

159. Aiken CB. Pramipexole in psychiatry: a systematic review of the literature. J Clin Psychiatry. 2007;68:1230-6.

160. Jaskiw GE, Popli AP. A meta-analysis of the response to chronic L-Dopa in patients with schizophrenia: therapeutic and heuristic implications. Psychopharmacology (Berl). 2004;171:365-74.

161. Kelleher JP, Centorrino F, Huxley NA, Bates JA, Drake JK, Egli S, et al. Pilot randomized, controlled trial of pramipexole to augment antipsychotic treatment. Eur Neuropsychopharmacol. 2012;22:415-8.

162. Di Milia L, Waage S, Pallesen S, Bjorvatn B. Shift work disorder in a random population sample-prevalence and comorbidities. PLoS ONE. 2013;8:e55306.

163. Paine SJ, Fink J, Gander PH, Warman GR. Identifying advanced and delayed sleep phase disorders in the general population: a national survey of New Zealand adults. Chronobiol Int. 2014;31:627-36.

164. Morgenthaler TI, Lee-Chiong T, Alessi C, Friedman L, Aurora RN, Boehlecke B, et al. Standards of Practice Committee of the American Academy of Sleep Medicine. Practice parameters for the clinical evaluation and treatment of circadian rhythm sleep disorders. An American Academy of Sleep Medicine report. Sleep. 2007;30:1445-59.

165. De Crescenzo F, Lennox A, Gibson JC, Cordey JH, Stockton S, Cowen PJ, et al. Melatonin as a treatment for mood disorders: a systematic review. Acta Psychiatr Scand. 2017;136:549-58.

166. Igwe SC, Brigo F. Does melatonin and melatonin agonists improve the metabolic side effects of atypical antipsychotics?: a systematic review and metaanalysis of randomized controlled trials. Clin Psychopharmacol Neurosci. 2018;16:235-245. 